\title{
THE $L^{p}$ NORM OF SUMS OF TRANSLATES OF A FUNCTION
}

\author{
BY \\ MAREK KANTER
}

ABSTRACT. For $p$ not an even integer, $p>0$, we prove that knowledge of the $L^{p}$ norm of all linear combinations of translates of a real valued function in $L^{p}(R)$ determines the function up to translation and multiplication by \pm 1 .

1. Introduction. Let $R$ stand for the real line, let $p$ be a real number greater than zero, and let $L^{p}(R)$ stand for the set of all real valued Borel measurable functions $f$ on $R$ such that $\int_{R}|f(s)|^{p} d s$ is finite. We denote $\left(\int_{R}|f(s)|^{p}\right)^{1 / p}$ by $\|f\|_{p}$. We denote the function $s \rightarrow f(t+s)$ by $f^{(t)}$. We denote [f] the set of all functions $g$ in $L^{p}(R)$ such that $\left\|g \pm f^{(t)}\right\|_{p}=0$ for some $t \in R$.

We note that for $g \in[f]$ we have

$$
\left(\left\|a_{0} f^{\left(t_{0}\right)}+\ldots+a_{n} f^{\left(t_{n}\right)}\right\|_{p}\right)^{p}=\left(\left\|a_{0} g^{\left(t_{0}\right)}+\ldots+a_{n} g^{\left(t_{n}\right)}\right\|_{p}\right)^{p}
$$

for all numbers $a_{0}, \ldots, a_{n}$ in $R$ and all points $t_{0}, \ldots, t_{n}$ in $R$.

Conversely, suppose we know the functions

$$
\phi_{n}\left(a_{0}, \ldots, a_{n}, t_{0}, \ldots, t_{n}\right) \equiv\left(\left\|a_{0} f^{\left(t_{0}\right)}+\ldots+a_{n} f^{\left(t_{n}\right)}\right\|_{p}\right)^{p}
$$

Does this knowledge determine the equivalence class [f]? (I.e., is $f$ determined up to translation and multiplication by \pm 1 ?)

Our question has a negative answer in the case $p=2$. In that case knowledge of $\left\{\phi_{n}\right\}$ is equivalent to knowledge of the function $r(t) \equiv \int_{R} f(t+s) f(s) d s$ for all $t \in R$. However, it is well known that knowledge of $r(t)$ does not determine $f$ as above. (See Doob [2, p. 586] for a complete description of what else is needed in the case $p=2$ to determine $[f]$.)

In this paper we show that, in fact, whenever $p$ is not an even integer, then knowing the functions $\phi_{n}$ determines [f]. We presume that this result is false whenever $p$ is any even integer; however the only counterexamples we have at the moment are for $p=2$.

Our proof is interesting in that it uses the concepts of integration in infinite dimensional spaces in order to prove a result that seems classical. The proof hinges on a lemma concerning symmetric measures on $S^{n}$, the $n$-dimensional surface of a sphere. Namely, if $\mu$ is such a measure then knowing

$$
\phi(\alpha)=\int_{S^{n}}|(\alpha \cdot e)|^{p} d \mu(e)
$$

Received by the editors January 5, 1972.

AMS (MOS) subject classifications (1970). Primary 43A15, 43A85, 46E30; Secondary 60G05, 60G10, 62Ml5, 28A40.

Key words and phrases. Fourier transform, $L^{p}$ norm of translates, measures on spheres. 
for all $\alpha \in R^{n+1}$ determines $\mu$, if $p$ is not even. (By the way, for $p$ any even integer this lemma is false. Say $n=1$. We are then asking whether knowing $\phi(t)$ $=\int_{[0, \pi]}|\cos (x+t)|^{p} d \mu(x)$ for all $t$ determines the measure $\mu$. This is false because the Fourier series of $|\cos x|^{p}$ has only finitely many nonzero terms whenever $p$ is even.)

The lemma concerning measures on spheres is due to Paul Levy for $0<p$ $<2$. We have used its proof in Bochner [1] to generalize it.

Let us end this introduction by relating our motivation for this work. Namely, if $0<p<2$ then there exists a time homogeneous symmetric stable stochastic process $X$ of index $p$ with independent increments. If $f$ is in $L^{p}(R)$ then we can consider the stationary process $Y(t)$ formed by letting $Y(t)=\int_{R} f(t+s) d X(s)$. (We refer the reader to [6] for an elementary account of stable random variables and stochastic integrals such as above.) The results of this paper imply, in particular, that knowing the finite dimensional joint distributions of the process $Y$ determines $f$ as above. We hope to exploit this result for stochastic processes in a further paper.

\section{Symmetric measures on spheres.}

Notation. Let $n$ be any nonnegative integer. If $x=\left(x_{0}, \ldots, x_{n}\right)$ is a point in $R^{n+1}$ we set $\|x\|_{p}=\left(\sum_{i=0}^{n}\left|x_{i}\right|^{p}\right)^{1 / p}, S^{n}=\left\{e \mid e \in R^{n+1},\|e\|_{2}=1\right\}$. For $\alpha, e \in R^{n+1}$ we define $(\alpha \cdot e)=\sum_{i=0}^{n} \alpha_{i} e_{i}$. Finally for $r \in(0, \infty), x \in R^{n+1}$, let $\theta(r, x)=r x$.

Lemma 1. Suppose $\mu$ is a symmetric bounded measure on $S^{n}$. ( $\mu$ symmetric means $\mu(A)=\mu(-A)$ for any Borel subset of $S^{n}$.) Suppose $m$ is any positive integer and suppose that $\rho$ is a positive measure on $(0, \infty)$ such that

$$
\begin{gathered}
\int_{0}^{1} r^{2 m} d \rho(r)<\infty, \\
\int_{1}^{\infty} r^{2(m-1)} d \rho(r)<\infty .
\end{gathered}
$$

Let us define

$$
\psi(w)=\int_{0}^{\infty}\left(\cos (w r)-1+\frac{(w r)^{2}}{2}-\ldots+\frac{(-1)^{m-1}}{(2(m-1)) !}(w r)^{2(m-1)}\right) d \rho(r)
$$

for every $w \in R$. (Note that the integral defining $\psi$ exists by (1) and (2).)

We claim that knowing $\phi(\alpha)=\int_{S^{n}} \psi(\alpha \cdot e) d \mu(e)$ for all $\alpha$ in $R^{n+1}$ determines $\mu$.

Proof. Let us first prove the lemma for nonnegative measures $\mu$. If $f(v)$ is a real valued function on $R$ and $h=\left(h_{0}, \ldots, h_{n}\right)$, define the function

$$
D_{h}(f)(\alpha)=\frac{1}{4}\left(\sum_{j=0}^{n} f\left(\alpha+h_{j} \delta_{j}\right)-2 f(\alpha)+f\left(\alpha-h_{j} \delta_{j}\right)\right)
$$


where $\delta_{j}=(0, \ldots, 0,1,0, \ldots, 0)$ and the 1 is at the $j$ th coordinate. Define $D_{h}^{2}(f)$ to be $D_{h}\left(D_{h}(f)\right)$, and for every integer $k \geq 2$ define $D_{h}^{k}(f)=D_{h}\left(D_{h}^{k-1} f\right)$.

Let $F$ stand for the measure $\rho \times \mu$ on $(0, \infty) \times S^{n}$, and define the measure $G$ on $R^{n+1}$ by setting $G(A)=F\left(\theta^{(-1)}(A)\right)$ for any Borel subset $A$ of $R^{n+1}$. We then have

$$
\begin{aligned}
\phi(\alpha)=\int_{R^{n+1}} & \left(\cos (\alpha \cdot x)-1+\frac{(\alpha \cdot x)^{2}}{2}\right. \\
& \left.\quad \ldots+\frac{(-1)^{m-1}}{(2(m-1)) !}(\alpha \cdot x)^{2(m-1)}\right) d G(x)
\end{aligned}
$$

Now let us note that

$$
D_{h}^{m} \phi(\alpha)=\int_{R^{n+1}} D_{h}^{m}(\cos (\alpha \cdot x)) d G(x)
$$

because $D_{h}^{n}\left((\alpha \cdot x)^{k}\right)=0$ for any integer $k<2 m$. Furthermore, it is not hard to see that

$$
D_{h}^{m}(\cos (\alpha \cdot x))=\left(-\sum_{j=0}^{n} \sin ^{2}\left(\frac{1}{2} h_{j} x_{j}\right)\right)^{m} \cos (\alpha \cdot x)
$$

and that

$$
D_{h}^{m}(\sin (\alpha \cdot x))=\left(-\sum_{j=0}^{n} \sin ^{2}\left(\frac{1}{2} h_{j} x_{j}\right)\right)^{m} \sin (\alpha \cdot x) .
$$

Noting now that $\left(\sum_{j=0}^{n} \sin ^{2}\left(\frac{1}{2} h_{j} x_{j}\right)\right)^{m}$ is $G$ integrable and that

$$
\int_{R^{n+1}}\left(\sum_{j=0}^{n} \sin ^{2}\left(\frac{1}{2} h_{j} x_{j}\right)\right)^{m} \sin (\alpha \cdot x) d G(x)=0
$$

because $\mu$ is symmetric, we write

$$
D_{h}^{m} \phi(\alpha)=\int_{R^{n+1}} e^{i(\alpha \cdot x)}\left(-\sum_{j=0}^{n} \sin ^{2}\left(\frac{1}{2} h_{j} x_{j}\right)\right)^{m} d G(x) .
$$

By the well-known uniqueness property of the Fourier transform of a finite measure, we conclude that the finite measure $\left(\sum_{j=0}^{n} \sin ^{2}\left(\frac{1}{2} h_{j} x_{j}\right)\right)^{m} d G(x)$ is determined for all $h=\left(h_{0}, \ldots, h_{n}\right)$. It follows that the measure $G$ is determined on the set

$$
A_{h}=\left\{x \mid x=\left(x_{0}, \ldots, x_{n}\right), \sum_{j=0}^{n} \sin ^{2}\left(\frac{1}{2} h_{j} x_{j}\right)>0\right\}
$$


We can choose $h=\left(h_{0}, \ldots, h_{n}\right)$ and $h^{\prime}=\left(h_{0}^{\prime}, \ldots, h_{n}^{\prime}\right)$ such that $A_{h} \cup A_{h^{\prime}}=R^{n+1}$ $-\{\overline{0}\}$, where $\overline{0}=(0, \ldots, 0) \in R^{n+1}$. It follows that $G$ is determined on $R^{n+1}-\overline{0}$. But $G$ has no mass at $\overline{0}$; hence the measure $G$ is determined. Noting that $\theta$ is $1-1$, we conclude that the measure $F$ is determined. Now $F=\rho \times \mu$, and $\rho$ is fixed; hence $\mu$ is determined. This proves the lemma for $\mu$ nonnegative. If we only assume $\mu$ bounded, then $\mu$ can be written uniquely as $\mu_{1}-\mu_{2}$, where $\mu_{1}$ and $\mu_{2}$ are nonnegative. Applying the lemma to $\mu_{1}$ and $\mu_{2}$, we are done. Q.E.D.

The above lemma and its proof are an extension of the arguments in Bochner $[1$, p. 68] where it is proved for $0<p<2$.

Corollary 1. Let $p$ be any positive real number which is not an even integer. Then if $\mu$ is any bounded symmetric measure on $S^{n}$, knowledge of $\phi(\alpha)=$ $\int_{S^{n}}|(\alpha \cdot e)|^{p} d \mu(e)$ for all $\alpha$ in $R^{n+1}$ determines $\mu$.

Proof. Let $m$ be the unique positive integer such that $2(m-1)<p<2 m$.

Let $d \rho(r)=\left(1 / r^{1+p}\right) d r$ on $(0, \infty)$. Then $\rho$ satisfies conditions $(1)$ and $(2)$ of Lemma 1. Now form the function

$$
\psi(w)=\int_{0}^{\infty}\left(\cos (w r)-1+(w r)^{2}-\ldots+\frac{(-1)^{m-1}}{(2(m-1)) !}(w r)^{2(m-1)}\right) d \rho(r) .
$$

By a change of variables $r=a r^{\prime}$, we see that $\psi(a w)=a^{p} \psi(w)$ for all positive $a$. It follows that $\psi(w)$ equals $\pm 1\left(k_{p}\right)|w|^{p}$ for some positive constant $k_{p}$. Now apply Lemma 1. Q.E.D.

We wish to generalize Corollary 1 to measures $\mu$ which are not necessarily bounded. The technique of proof for this is similar to the technique in Lemma 1, but needs a certain amount of additional argumentation. Our first preliminary is the following:

Lemma 2. Let $m$ be any positive integer. Let the numbers $a_{1}, \ldots, a_{m}$ be defined by the equation

$$
(\cos x-1)^{m}=(-1)^{m}+\sum_{k=1}^{m} a_{k} \cos (k x) .
$$

Then for $p \in[2(m-1), 2 m)$ the sum $\sum_{k=1}^{m} a_{k} k^{p}$ vanishes only at the point $p=2(m-1)$.

Proof. $\left.\left(d^{2(m-1)} / d x^{2(m-1)}\right)(\cos x-1)^{m}\right|_{0}=(-1)^{m-1}\left(\sum_{k=1}^{m} a_{k} k^{2(m-1)}\right)$. Also it is clear that $\left.\left(d^{2(m-1)} / d x^{2(m-1)}\right)(\cos x-1)^{m}\right|_{0}=0$ because the Taylor series of $(\cos x$ - 1) starts with the term $-x^{2} / 2$. Hence the sum vanishes at the point $p$ $=2(m-1)$.

To prove the sum vanishes nowhere else on $[2(m-1), 2 m)$, let us consider

$$
\psi(w)=\int_{0}^{\infty}(\cos w r-1)^{m} \frac{1}{r^{1+p}} d r
$$


Arguing as in Corollary 1, we see that

$$
\psi(w)=(-1)^{m} K_{p}|w|^{p}
$$

for some positive constant $K_{p}$.

Let $S^{0}=\{-1,+1\}$ and let $\mu$ assign mass $\frac{1}{2}$ to each point in $S^{0}$. Let $\rho$ stand for the measure $d r / r^{1+p}$ on $(0, \infty)$. Let $F$ stand for the measure $\rho \times \mu$ on $(0, \infty) \times S^{0}$ and let $G=F \theta^{(-1)}$ as in Lemma 1 . We then have for all $\alpha \in R$

$$
\phi(\alpha)=(-1)^{m} K_{p}|\alpha|^{p}=\int_{R}(\cos (\alpha x)-1)^{m} d G(x) .
$$

Calculating as in Lemma 1 we can write for $h \in R$,

$$
D_{h}^{m} \phi(\alpha)= \pm\left(\frac{K_{p}}{k_{p}}\right) \int_{R} e^{i(\alpha x)}\left(-\sin ^{2}\left(\frac{1}{2} h x\right)\right)^{m} d G(x) .
$$

Let us now apply $D_{h}^{m}$ to $(\cos \alpha x-1)^{m}$. We note first that

$$
D_{h} \cos (\alpha k x)=-\cos (\alpha k x) \sin ^{2}\left(\frac{1}{2} k h x\right) .
$$

It follows that

$$
D_{h}^{m}(\cos (\alpha x)-1)^{m}=(-1)^{m} \sum_{k=1}^{m} a_{k} \cos (\alpha k x) \sin ^{2 m}\left(\frac{1}{2} k h x\right) .
$$

Let us now set $h=1$. It follows that

$$
\begin{aligned}
D_{1}^{m} \phi(\alpha) & =\int_{R} \sum_{k=1}^{m} a_{k} \cos (\alpha k x) \sin ^{2 m}\left(\frac{1}{2} k x\right) d G(x) \\
& =\sum_{k=1}^{m} a_{k} \int_{R} \cos (\alpha k x) \sin ^{2 m}\left(\frac{1}{2} k x\right) d G(x)
\end{aligned}
$$

Now we can rewrite

$$
\int_{R} \cos (\alpha k x) \sin ^{2 m}\left(\frac{1}{2} k x\right) d G(x)=k^{p} \int_{R} \cos (\alpha y) \sin ^{2 m}\left(\frac{1}{2} y\right) d G(y)
$$

by the change of variables $y=k x$ (and remembering that $d G(x)=\frac{1}{2} d x / x^{1+p}$ for $x>0$, and $d G(x)=\frac{1}{2} d x /|x|^{1+p}$ for $\left.x<0\right)$.

We can conclude that

$$
D_{1}^{m} \phi(\alpha)=\int_{R} \cos (\alpha y)\left(\sum_{k=1}^{m} a_{k} k^{p}\right) \sin ^{2 m}\left(\frac{1}{2} y\right) d G(y) .
$$

If the sum $\sum_{k=1}^{m} a_{k} k^{p}$ vanishes for some $p_{0} \in(2(m-1), 2 m)$, then we conclude that

$$
0=\int_{R} e^{i(\alpha x)}\left(\sin ^{2 m}\left(\frac{1}{2} x\right)\right) d G(x)
$$


where $d G(x)=\frac{1}{2} d x /|x|^{1+p_{0}}$. But this is clearly false. Q.E.D.

Now we are ready to generalize Corollary 1 to unbounded measures. We will at the same time extend the validity of the corollary to infinite dimensions and to surfaces more general than sphere surfaces. We start with some notation.

Let $Q$ stand for the rationals. For $p>0$, let $l_{Q}^{p}=\left\{\left.f\left|f \in R^{Q}, \sum_{t \in Q}\right| f(t)\right|^{p}\right.$ $<\infty\}$. For $\alpha \in l_{Q}^{p}$ and $f \in R^{Q}$ we define

$$
\begin{aligned}
(\alpha \cdot f) & =\sum_{t \in Q} \alpha(t) f(t) & & \text { if the sum converges, } \\
& =0 & & \text { if the sum does not converge. }
\end{aligned}
$$

Let $\mathcal{B}_{Q}$ denote the Borel $\sigma$-field generated by the product topology of $R^{Q}$. For $r \in(0, \infty)$ and $f \in R^{Q}$ define $\theta(r, f)=r f$. If $\mathcal{B}$ denotes the Borel subsets of $(0, \infty)$ then it is clear that $\theta^{(-1)}\left(\mathcal{B}_{Q}\right) \subset \mathcal{B} \times \mathcal{B}_{Q}$.

Lemma 3. Fix $p \in(0, \infty)$ and assume that $p$ is not an even integer. Let $S$ be a Borel subset of $R^{Q}$ and suppose that $\theta$ restricted to $(0, \infty) \times S$ is a 1-1 map into $R^{Q}$. Suppose also that $S$ is symmetric, i.e. $f \in S$ iff $-f \in S$. Let $\mu$ stand for a $\sigma$-finite measure on $\mathcal{B}_{Q}$ with values in $[0, \infty]$ such that $\mu$ is symmetric $(i . e . \mu(A)=\mu(-A)$ for $\left.A \in \mathcal{B}_{Q}\right)$ and such that:

(1) $\mu$ lives on $S$, i.e. $\mu\left(R^{Q}-S\right)=0$.

(2) Letting $p^{\prime}=p \wedge 1=\min (1, p)$, then for all $\alpha \in l_{Q}^{p^{\prime}}$ we have $\phi(\alpha)=$ $\int_{S}|(\alpha \cdot e)|^{p} d \mu(e)<\infty$ and $\sup _{t \in Q} \phi\left(\delta_{t}\right)=M<\infty$, where $\delta_{t} \in R^{Q}$ and $\delta_{t}(s)=0$ if $s \neq t$, and $\delta_{t}(t)=1$.

(3) $\mu\left(\cap_{t \in Q} B_{t}^{a}\right)=0$ for some $a>0$, where $B_{t}^{a}=\{e \mid e \in S$,e(t) is an integer multiple of $a \pi\}$.

Then $\mu$ is uniquely determined on $\dot{B}_{Q}$ by knowing $\phi$.

Proof. Let $m$ be the unique positive integer such that $2(m-1)<p<2 m$.

Let $\mathcal{B}_{S}$ stand for $\left\{A \mid A \in \mathcal{B}_{Q}, A \subset S\right\}$. Let $\rho$ stand for the measure $d r / r^{1+p}$ on $((0, \infty), \mathcal{B})$ and let $F$ stand for the measure $\rho \times \mu$ on $\left((0, \infty) \times S, \mathcal{B} \times \mathcal{B}_{S}\right)$. Since $\theta$ is a measurable map we can define the measure $G=F \theta^{(-1)}$ on $\left(R^{Q}, \mathcal{B}_{Q}\right)$.

Let us remember that by Lemma 2

$$
\int_{0}^{\infty}(1-\cos (r w))^{m} d \rho(r)=K_{p}|w|^{p}
$$

It follows, using Fubini's theorem, that we can write

$$
K_{p} \int_{S}|(\alpha \cdot e)|^{p} d \mu(e)=\int_{R^{Q}}(1-\cos (\alpha \cdot f))^{m} d G(f)
$$

for $\alpha \in l_{Q}^{\prime}$. Now $(1-\cos (\alpha \cdot f))=2 \sin ^{2}\left(\frac{1}{2} \alpha \cdot f\right)$ so we conclude that $\left(\sin ^{2}\left(\frac{1}{2}\right) \alpha \cdot f\right)^{m}$ is $G$ integrable for all $\alpha \in l_{Q}^{p^{\prime}}$. Remembering $M=\sup _{t \in Q} \phi\left(\delta_{t}\right)<\infty$, we can further write for $\alpha=(\alpha(t), t \in Q), \alpha \in l_{Q}^{p^{\prime}}$, 


$$
\begin{aligned}
\left(\int_{R^{Q}}(\right. & \left.\left.\sum_{t \in Q} \sin ^{2}\left(\frac{1}{2} \alpha(t) f(t)\right)^{m}\right) d G(f)\right)^{1 / m} \\
& \leq \sum_{t \in Q}\left(\int_{R^{Q}}\left(\sin ^{2 m}\left(\frac{1}{2} \alpha(t) f(t)\right)\right) d G(f)\right)^{1 / m} \\
& =\frac{1}{2} \sum_{t \in Q}\left(\int_{R^{Q}}(1-\cos (\alpha(t) f(t)))^{m} d G(f)\right)^{1 / m} \\
& =\frac{1}{2} K_{p}^{1 / m} \sum_{t \in Q}\left(\int_{S}|\alpha(t) e(t)|^{p} d \mu(e)\right)^{1 / m} \\
& =\frac{1}{2} K_{p}^{1 / m} \sum_{t \in Q}|\alpha(t)|^{p / m}\left(\phi\left(\delta_{t}\right)\right)^{p / m} \\
& \leq\left(\frac{1}{2} K_{p}^{1 / m} M^{p / m}\right) \sum_{t \in Q}|\alpha(t)|^{p / m} .
\end{aligned}
$$

If $p>2$ then $p / m>1$; hence $\sum_{t \in Q}|\alpha(t)|^{p / m}<\infty$, because $p^{\prime}=1$ and $\alpha \in l_{Q}^{p^{\prime}}$. If $0<p<2$, then $m=1$ and $\sum_{t \in Q}|\alpha(t)|^{p}<\infty$.

We conclude that $\left(\sum_{t \in Q} \sin ^{2}\left(\frac{1}{2} \alpha(t) f(t)\right)\right)^{m}$ is $G$ integrable for all $\alpha \in l_{Q}^{p^{\prime}}$.

Let us now take $h \in l_{Q}^{p^{\prime}}, \alpha \in l_{Q}^{p^{\prime}}$, and suppose $\{t \mid h(t) \neq 0\}$ is a finite set $N$. We define $D_{h} \phi(\alpha)$ to be

$$
\frac{1}{4} \sum_{t \in Q}\left\{\phi\left(\alpha+h(t) \delta_{t}\right)-2 \phi(\alpha)+\phi\left(\alpha-h(t) \delta_{t}\right)\right\} .
$$

We define $D_{h}^{2} \phi=D_{h}\left(D_{h} \phi\right)$, and for every integer $k \geq 2$ define $D_{h}^{k} \phi$ $=D_{h}\left(D_{h}^{k-1} \phi\right)$.

Now for an arbitrary $h \in l_{Q}^{p^{\prime}}$, let $N_{n}=\{t|| h(t) \mid \geq 1 / n\}$, and let $h_{n}(t)=h(t)$ if $t \in N_{n}$; otherwise let $h_{n}(t)=0$.

Let us define

$$
D_{h}^{m} \phi(\alpha)=\lim _{n \rightarrow \infty} D_{h_{n}}^{m} \phi(\alpha)
$$

To show this limit exists we use formula (a) of Lemma 1 to write

$$
K_{p} D_{h_{n}}^{m} \phi(\alpha)=\sum_{k=1}^{m} a_{k} \int_{R^{e}} \cos (k \alpha \cdot f)\left(\sum_{t \in N_{n}} \sin ^{2}\left(\frac{1}{2} k h(t) f(t)\right)\right)^{m} d G(f),
$$

where $a_{k}$ are as defined in Lemma 2.

Now $\left(\sum_{t \in Q} \sin ^{2}\left(\frac{1}{2} \alpha(t) f(t)\right)\right)^{m}$ is $G$ integrable; hence by the Lebesgue monotone convergence theorem $K_{p}\left(\lim _{n \rightarrow \infty} D_{h_{n}}^{m} \phi(\alpha)\right)$ exists and equals

$$
\sum_{k=1}^{m} a_{k} \int_{R^{Q}} \cos (k \alpha \cdot f)\left(\sum_{t \in Q} \sin ^{2}\left(\frac{1}{2} k h(t) f(t)\right)\right)^{m} d G(f) .
$$

By formula (b) of Lemma 2, we can rewrite this as

$$
\left(\sum_{k=1}^{m} a_{k} k^{p}\right) \int_{R^{Q}} \cos (\alpha \cdot f)\left(\sum_{t \in Q} \sin ^{2}\left(\frac{1}{2} h(t) f(t)\right)\right)^{m} d G(f) .
$$


Now Lemma 2 ensures us that the first factor does not vanish. It follows that knowing $\phi(\alpha)$ determines the function $\tau(\alpha)$ :

$$
\tau(\alpha)=\int_{R^{e}} \cos (\alpha \cdot f)\left(\sum_{t \in Q} \sin ^{2}\left(\frac{1}{2} h(t) f(t)\right)\right)^{m} d G(f) .
$$

Let us use the fact that $\mu$ is symmetric to rewrite $\tau(\alpha)$ as

$$
\int_{R^{Q}} e^{i(\alpha \cdot f)}\left(\sum_{t \in Q} \sin ^{2}\left(\frac{1}{2} h(t) f(t)\right)\right)^{m} d G(f)
$$

By the uniqueness property of Fourier transforms of finite measures, it is clear that the marginal distributions of the finite measure

$$
\left(\sum_{t \in Q} \sin ^{2}\left(\frac{1}{2} h(t) f(t)\right)\right)^{m} d G(f)
$$

are uniquely determined on all finite dimensional subspaces of $R^{Q}$. This implies we know this measure on $\mathcal{B}_{Q}$.

Let us now use (3) to pick $a>0$ such that $\mu\left(\cap_{t \in Q} B_{t}^{a}\right)=0$. Let $\left\{t_{0}, t_{1}, \ldots\right\}$ be a list of the members of $Q$. Pick $k$ to be an integer with $k p>1$ and let $h\left(t_{n}\right)=n^{-2 k} q^{(-1)}$. Then $h \in l_{Q}^{p^{\prime}}$. Also we have that $\sum_{t \in Q} \sin ^{2}\left(\frac{1}{2} h(t)\right) f(t)>0$ a.s. with respect to the measure $G$ by the Fubini theorem. We conclude that knowing the measure $(*)$ for this $h$ determines the measure $G$.

Let us remember that $\theta$ is a $1-1$ measurable map from $(0, \infty) \times S$ into $R^{Q}$. However, $(0, \infty) \times S$ is a Borel subset of a complete metric space. Hence, by Kuratowski [4, p. 397], we see that $\theta^{(-1)}\left(\mathcal{B}_{Q}\right) \in \mathcal{B} \times \mathcal{B}_{S}$. This implies the transformation that sends a measure $F$ on $\left((0, \infty) \times S, \mathcal{B} \times \mathcal{B}_{S}\right)$ to the measure $\theta^{(-1)} F$ on $\left(R^{Q}, \mathcal{B}_{Q}\right)$ is a $1-1$ transformation. Since we know $G=\theta^{(-1)} F$, we conclude that $F$ is uniquely determined. $F=\rho \times \mu$, and we conclude further that the measure $\mu$ is uniquely determined on $\mathcal{B}_{Q}$ by knowing $\phi$. Q.E.D.

3. The main results. Let us start with a finite dimensional analogue of our main theorem. Namely for $x=\left(x_{0}, x_{1}, \ldots, x_{n}\right) \in R^{n+1}$, let us define $x^{(0)}=x, x^{(1)}$ $=\left(x_{1}, x_{2}, \ldots, x_{n}, x_{0}\right), \ldots, x^{(n)}=\left(x_{n}, x_{0}, \ldots, x_{n-1}\right)$. For $\alpha=\left(\alpha_{0}, \ldots, \alpha_{n}\right)$ we can consider the function

$$
\phi(\alpha)=\left(\left\|\alpha_{0} x^{(0)}+\ldots+\alpha_{n} x^{(n)}\right\|_{p}\right)^{p} .
$$

Lemma 4. If $p \in(0, \infty)$ is not an even integer then the function $\phi$ determines the vector $x$ up to shift and multiplication by \pm 1 .

Proof. Let us first start by saying that if $x, y$ are points of $R^{n+1}$, then to say that $x$ and $y$ are equal up to shift and multiplication by \pm 1 means that for some $i \in\{0,1, \ldots, n\}$ we have $x^{(i)}= \pm y$, and in that case the corresponding functions $\phi$ will be the same. 
Let $x \in R^{n+1}$ be fixed and construct the function $\phi$ as above. Let $M=\|x\|_{2}$. Let $e^{(i)}=x^{(i)} / M$. Then $e^{(i)}$ is a point of $S^{n}$ for $i \in\{0,1, \ldots, n\}$. Let mass $M^{p}$ be assigned to each of the points in $\{0,1, \ldots, n\}$, and let $\mu$ stand for the measure on $S^{n}$ induced from the map $i \rightarrow e^{(i)}$. Define the symmetrized measure $\mu_{s}$ by $\mu_{s}(A)=\frac{1}{2}(\mu(A)+\mu(-A))$ for any Borel subset of $S^{n}$.

It is easy to compute that

$$
\phi(\alpha)=\int_{S^{n}}|(\alpha \cdot e)|^{p} d \mu_{s}(e)
$$

for all $\alpha \in R^{n+1}$. By Corollary 1, the measure $\mu_{s}$ is determined. By the construction of $\mu_{s}$ this implies that the vector $x$ is determined up to shift and multiplication by \pm 1. Q.E.D.

We will now use Lemma 3 and an extension of the technique of Lemma 4 to prove our main result. One last notational preliminary: We let $\mathcal{L}_{0}(R)$ denote the set of all real valued functions $\alpha$ defined on $R$ with $\alpha(t)=0$ for all but a finite number of $t$.

Theorem 1. If $p \in(0, \infty)$ is not an even integer and $f_{0} \in L^{p}(R)$ then if we know for all $\alpha \in \mathcal{L}_{0}(R)$ the function $\phi(\alpha)$ defined by

$$
\phi(\alpha)=\left(\left\|\alpha\left(t_{0}\right) f_{0}^{\left(t_{0}\right)}+\ldots+\alpha\left(t_{n}\right) f_{0}^{\left(t_{n}\right)}\right\|_{p}\right)^{p}
$$

(where $\left\{t_{0}, \ldots, t_{n}\right\}=\{t \mid \alpha(t) \neq 0\}$ ), then we know $\left[f_{0}\right]$.

Proof. We treat $L^{p}(R)$ as a non-Hausdorff topological space. (We set $p^{\prime}$ $=p \wedge 1$ and then $\left(\|\|_{p}\right)^{p^{\prime}}$ is a pseudonorm on $L^{p}(R)$.)

We endow $L^{p}(R)$ with the Borel structure generated by its metric topology, and we consider the measurable map $t \rightarrow f_{0}^{(t)}$ from $R$ to $L^{p}(R)$. We let $\hat{\mu}$ be the measure on $L^{p}(R)$ induced by this map, i.e. $\hat{\mu}(A)=\left|\left\{t \mid f_{0}^{(t)} \in A\right\}\right|$ for any Borel set $A$ of $L^{p}(R)$. (For a Borel subset $B$ of $R$ we let $|B|$ stand for its Lebesgue measure.) We define

$$
\hat{\mu}_{s}(A)=\frac{1}{2}(\hat{\mu}(A)+\hat{\mu}(-A)) .
$$

It is clear that the symmetric measure $\hat{\mu}_{s}$ assigns measure zero to $L^{p}(R)-\left[f_{0}\right]$. Furthermore let us note that $\left[f_{0}\right]$ is a closed subset of $L^{p}(R)$ and that if $C$ is a closed subset of $\left[f_{0}\right]$ with $\left[f_{0}\right]-C \neq \varnothing$, then

$$
\hat{\mu}_{s}\left(\left[f_{0}\right]-C\right)=\frac{1}{2}\left(\left|\left\{t \mid f_{0}^{(t)} \notin C\right\}\right|+\left|\left\{t \mid f_{0}^{(t)} \notin-C\right\}\right|\right) .
$$

Now the function $t \rightarrow f_{0}^{(t)}$ is a continuous map; hence both $\left\{t \mid f_{0}^{(t)} \notin C\right\}$ and $\left\{t \mid f_{0}^{(t)} \notin-C\right\}$ are open subsets of $R$. It is easily seen that both of these open sets cannot be empty; hence, since the Lebesgue measure of a nonempty open set is strictly greater than zero, we conclude that $\hat{\mu}_{s}\left(\left[f_{0}\right]-C\right)>0$. In other words, $\left[f_{0}\right]$ is the support of $\hat{\mu}_{s}$ (i.e., $\left[f_{0}\right]$ is the smallest closed set containing all the mass of $\left.\hat{\mu}_{s}\right)$. 
Another fact which is clear is that if we had started with a different function $f_{1}$ with $\left[f_{1}\right]=\left[f_{0}\right]$, then the construction just described would have yielded the same measure $\hat{\mu}_{s}$.

We let $A$ denote the $\sigma$-field of subsets of $L^{p}(R)$ generated by finite unions of sets $B \cap A_{1} \ldots A_{n}$ where $A_{i}$ is a set of the form $\left\{g \mid g \in L^{p}(R), g\left(t_{i}\right) \in D_{i}\right\}$ (for $t_{i} \in Q$ and $D_{i}$ a Borel subset of $R$ ) and where $B$ is a Borel subset of $L^{p}(R)$. We can extend $\hat{\mu}_{s}$ to $\mathcal{A}$ by defining $\hat{\mu}\left(B \cap A_{1} \cap \ldots \cap A_{n}\right)=\mid\left\{t \mid f_{0}^{(t)} \in B \cap A_{1}\right.$ $\left.\cap \ldots \cap A_{n}\right\} \mid$, and then symmetrizing as before. We then let $\mathcal{A}_{Q}$ denote the $\sigma$ field generated by finite unions of sets $A_{1} \cap \ldots \cap A_{n}$ as above, and we let $\mathcal{A}_{Q}^{*}$ denote the $\hat{\mu}_{s}$ completion of $\mathcal{A}_{Q}$.

We assert that knowing $\mu_{s}$ on $\mathcal{A}_{Q}^{*}$ determines $\mu_{s}$ on $\mathcal{A}$. To prove this assertion we write, for $f$ in $L^{p}(R)$,

$$
\begin{aligned}
\left(\|f\|_{p}\right)^{p} & =\int_{-\infty}^{+\infty}|f(s)|^{p} d s=\sum_{m=-\infty}^{+\infty} \int_{[0,1]}|f(s+m)|^{p} d s \\
& =\int_{[0,1]} \sum_{m}|f(s+m)|^{p} d s .
\end{aligned}
$$

We conclude that $\sum_{m}|f(s+m)|^{p}<\infty$ a.e. in $[0,1]$ for any $f \in L^{p}(R)$. Of course we could have used any other interval $[k, k+1]$ instead of $[0,1]$. So we have $\sum_{m}|f(s+m)|^{p}<\infty$. Let us define

$$
f^{*}(s)=\sum_{m}|f(s+m)|^{p}
$$

if the sum converges. $f^{*}(s)=0$ if the sum diverges.

It is clear that $f^{*}$ is periodic of period 1 and also that $\int_{0}^{1} f^{*}(s) d s=\left(\|f\|_{p}\right)^{p}$. Let us define

$$
Q(f)=\lim _{n \rightarrow \infty} 2^{-n}\left[\sum_{k=0}^{2^{n}-1} f^{*}\left(k / 2^{-n}\right)\right]
$$

if the limit exists and 0 otherwise. By a martingale type argument as given in Hewitt [7, p.375] we conclude that $Q\left(f^{(t)}\right)=\left(\|f\|_{p}\right)^{p}$ a.e. in $R$.

If we apply the above to $f_{0}$, we can conclude that for $g_{1}, \ldots, g_{n}$ in $L^{p}(R)$ and numbers $k_{1}, \ldots, k_{n}$ all $>0$ we have $\mu_{s}(B)=\mu_{s}(D)$ where $B=\bigcap_{i=1}^{n} B_{k_{i}}\left(g_{i}\right)$, $D=\bigcap_{i=1}^{n} D_{k_{i}}\left(g_{i}\right)$, and $B_{k_{i}}\left(g_{i}\right)=\left\{f \mid f \in L^{p}(R),\left\|f-g_{i}\right\|_{p}<k_{i}\right\}$ while $D_{k}\left(g_{i}\right)$ $=\left\{f \mid f \in L^{p}(R), Q\left(f-g_{i}\right)<k_{i}^{p}\right\}$. Knowing $\mu_{s}$ on sets of the form $B$ clearly determines $\mu_{s}$ on $\mathcal{A}$, while sets of the form $D$ are certainly in $\mathcal{A}_{Q}^{*}$. We conclude that knowing $\mu_{s}$ on $\mathcal{A}_{Q}^{*}$ determines $\mu_{s}$ on $\mathcal{A}$.

We are now ready to use Lemma 3 . Let $S=\left\{e \mid e \in R^{Q}\right.$ and $e$ satisfies (*)\}:

$$
Q(e)=\left(\left\|f_{0}\right\|_{p}\right)^{p}
$$

It is clear that $\theta$ as defined in Lemma 3 is a $1-1$ map from $(0, \infty) \times S$ into $R^{Q}$, unless $\left\|f_{0}\right\|_{p}=0$, which we exclude as a trivial case. Let us define the map 
$I: L^{p}(R) \rightarrow R^{Q}$ by setting $I(g)(t)=g(t)$ for $g \in L^{p}(R)$ and $t \in Q$. By what we have already shown it follows that we can throw away an $A$ measurable set $A$ of $\hat{\mu}_{s}$ measure zero from $L^{p}(R)$ such that

$$
I\left(L^{p}(R)-A\right) \subset S
$$

Defining $\mathcal{B}_{S}$ as in Lemma 3, it is clear that $I^{(-1)}\left(\mathcal{B}_{S}\right) \subset \mathcal{A}_{Q}$. We denote by $\mu$ the measure induced on $\left(S, \mathcal{B}_{S}\right)$ by setting $\mu(B)=\hat{\mu}_{s}\left(I^{(-1)}(B)\right)$ for $B \in \mathcal{B}_{S}$. It is clear that $S$ is a symmetric subset of $R^{Q}$ and that $\mu$ is a symmetric measure. We now need to check that hypotheses (1) through (3) of Lemma 3 are satisfied.

(1) is obvious from $(* *)$.

(2) To prove (2) let us consider $\alpha \in \mathcal{L}_{0}(R)$ with $\left\{t_{0}, \ldots, t_{n}\right\}=\{t \mid \alpha(t) \neq 0\}$

$\subset Q$. We have

$$
\begin{aligned}
\int_{S}|(\alpha \cdot e)|^{p} d \mu_{s}(e) & =\int_{S}\left|\alpha\left(t_{0}\right) e\left(t_{0}\right)+\ldots+\alpha\left(t_{n}\right) e\left(t_{n}\right)\right|^{p} d \mu(e) \\
& =\int_{R}\left|\alpha\left(t_{0}\right) f_{0}\left(t_{0}+s\right)+\ldots+\alpha\left(t_{n}\right) f_{0}\left(t_{n}+s\right)\right|^{p} d s \\
& =\left(\left\|\alpha\left(t_{0}\right) f_{0}^{\left(t_{0}\right)}+\ldots+\alpha\left(t_{n}\right) f_{0}^{\left(t_{n}\right)}\right\|_{p}\right)^{p}
\end{aligned}
$$

We conclude that the function $\phi$ of Theorem 1 coincides with the function $\phi$ of Lemma 3 at least on $\mathcal{L}_{0}(R)$.

Let us now define $\mathcal{L}_{Q}^{p}$ to be the set of all real valued functions $\alpha$ defined on $R$ with $\{t \mid \alpha(t) \neq 0\} \subset Q$ and also $\sum_{Q}|\phi(t)|^{p}<\infty$.

We can identify $l_{Q}^{p}$ with $\mathcal{L}_{Q}^{p}$. We let $p^{\prime}=p \wedge 1$. We have for $\alpha \in l_{Q}^{p^{\prime}}$,

$$
\begin{aligned}
\int|(\alpha \cdot e)|^{p} d \mu_{s}(e) & \leq \int_{S} \sum_{t \in Q}|\alpha(t) e(t)|^{p} d \mu_{s}(e) \\
& =\left(\|f\|_{p}\right)^{p}\left(\sum_{t \in Q}|\alpha(t)|^{p}\right)<\infty .
\end{aligned}
$$

If we let $\left\{t_{0}, t_{1}, \ldots\right\}$ be a list of the elements of $Q$ and if we define

$$
\begin{aligned}
\alpha_{n}(t) & =\alpha(t) & & \text { if } t \in\left\{t_{0}, \ldots, t_{n}\right\}, \\
& =0 & & \text { if not, }
\end{aligned}
$$

then

$$
\int\left|\left(\left(\alpha-\alpha_{n}\right) \cdot e\right)\right|^{p} d \mu_{s}(e) \leq\left(\|f\|_{p}\right)^{p}\left(\sum_{k=n+1}^{\infty}\left|\alpha\left(t_{k}\right)\right|^{p}\right) .
$$

It follows that we can define $\phi(\alpha)=\lim _{n \rightarrow \infty} \phi\left(\alpha_{n}\right)$ and we will have

$$
\phi(\alpha)=\int_{S}|(\alpha \cdot e)|^{p} d \mu_{s}(e)
$$


for $\alpha \in l_{Q}^{p^{\prime}}$.

Now $\sup _{t \in Q} \phi(t)=\sup _{t \in Q}\left(\left\|f_{\delta}^{(t)}\right\|_{p}\right)^{p}=\left(\|f\|_{p}\right)^{p}<\infty$, so (2) is satisfied.

To prove (3) we note that there must be some $a>0$ such that

$$
0=\mid\{t \mid f(t)=k \pi a \text { for some nonzero integer } k\} \mid
$$

(where $|A|$ stands for the Lebesgue measure of a Borel set $A$ in $R$ ). If the above were false for all $a>0$ then we would have that $R$ contained uncountably many disjoint sets of positive Lebesgue measure. So let us fix an $a>0$ for which the above is true, and let us consider the "complementary" set $B_{a}=\left\{t \mid f_{0}(t)\right.$ $\neq k \pi a$ for any integer $k\}$.

Now $|\{t \mid f(t) \neq 0\}|>0$; hence $\left|B_{a}\right|>0$. From this it is not hard to show that for Lebesgue a.e. $t$ we have $\left(B_{a}+t\right) \cap Q$ is nonempty. We conclude that (3) is satisfied.

From Lemma 3 we conclude that $\mu_{s}$ is uniquely determined on $\mathcal{B}_{s}$. It follows that $\hat{\mu}_{s}$ is uniquely determined on $I^{(-1)}\left(\mathcal{B}_{S}\right)$. However the $\hat{\mu}_{s}$ completion of $I^{(-1)}\left(B_{S}\right)$ is clearly $\mathcal{A}_{Q}^{*}$. It follows that $\hat{\mu}_{s}$ is uniquely determined on the Borel subsets of $L^{p}(R)$. In particular the support of $\hat{\mu}_{s}$ is uniquely determined, which in this case happens to be the closed set $\left[f_{0}\right]$.

However, knowing $\left[f_{0}\right]$ means we know $f_{0}$ up to shift in time and multiplication by \pm 1 . Q.E.D.

In the above proof we argued that $\mathcal{A}_{Q}^{*}$ contained the Borel subsets of $L^{p}(R)$. A similar but more general such argument can be found in Itô [3].

4. Generalizations. Let us formulate a general conjecture.

Conjecture. Let $p$ be any positive real number not of the form $2 n$. Let $G$ be any locally compact abelian group. Let $\gamma$ stand for Haar measure on $G$. Then if we know the $L^{p}$ norm of all linear combinations of a real valued function $f \in L^{p}(G, \gamma)$, then we know $f$ up to translation and multiplication by \pm 1 .

We have proved this conjecture when $G=R$. Our proof specializes and gives us the truth of this conjecture for $G=$ integers $\}$, and also $G=[0,1]$. Lemma 4 proves this conjecture for any finite abelian group $G$.

The critical point in extending Theorem 1 to any locally compact abelian group is to extend Lemma 5 to any compact abelian group. It is easy to see how to extend Lemma 5 to $G=[0,1] \times[0,1] \times \ldots \times[0,1]$; in other words we know the conjecture for $G$ any direct product of finitely many copies of $R$ and [0,1]. A little structure theory should finish the job.

Finally, the conjecture should be studied for general nonabelian locally compact groups.

Acknowledgement. I would like to thank Professor Robert M. Blumenthal of the University of Washington for several useful conversations concerning Lemma 1 .

Added in proof. We can extend our main theorem to complex $L^{p}$ spaces and, for $p$ not even, show that knowledge of the $L^{p}$ norm of all complex linear 
combinations of translates of a complex valued function in $L^{p}(R)$ determines the function up to translation and multiplication by $e^{i \theta}$ for some $\theta$ in $[0,2 \pi]$. The proof is essentially the same as in the real case, except that Corollary 1 has to be extended to complex $n$-space. We let $C$ stand for the complex numbers and we let

$$
S_{c}^{n}=\left\{\left.\left(z_{1}, \ldots, z_{n+1}\right)\left|z_{i} \in C, \sum_{1}^{n+1}\right| z_{i}\right|^{2}=1\right\} .
$$

If $\mu$ is a measure on $S_{c}^{n}$ we say it is complex symmetric if $\mu(A)=\mu\left(e^{i \theta} A\right)$ for all $\theta \in[0,2 \pi]$ and all Borel subsets $A$ of $S_{c}^{n}$. We say it is real symmetric if we only have $\mu(A)=\mu(-A)$.

Lemma 1'. Suppose $p$ is not even. Define $m, \rho$, and $\psi$ as in Lemma 1. If $\mu$ is a real symmetric measure on $S_{c}^{n}$ we claim that knowing

$$
\phi(\alpha)=\int_{S_{c}^{n}}(\operatorname{Re}(\alpha \cdot z)) d \mu(z)
$$

for all $\alpha$ in $C^{n+1}$ is sufficient to determine $\mu$.

The proof of this lemma is virtually the same as the proof of Lemma 1, except that we apply the operator $D_{h}^{m}$ to functions of the form $e^{i \operatorname{Re}(\alpha \cdot z)}$ and $\operatorname{Re}\left((\alpha \cdot z)^{k}\right)$. To apply Lemma $1^{\prime}$ to complex $L^{p}$ space we let $d \rho(r)=\left(1 / r^{1+p}\right) d r$. Then for $w \in C$ we have

$$
\pm k_{p}|w|^{p}=\int_{0}^{2 \pi} \psi\left(\operatorname{Re}\left(e^{i \theta} w\right) d \theta\right.
$$

for some positive constant $k_{p}$.

If $\mu$ is complex symmetric we can then conclude from Lemma $1^{\prime}$ that knowing $\int_{S_{c}^{n}}|\alpha \cdot z|^{p} d \mu(z)$ for all $\alpha \in C^{n+1}$ determines $\mu$.

\section{REFERENCES}

1. S. Bochner, Harmonic analysis and the theory of probability, Univ. of California Press, Berkeley, 1955. MR 17, 273.

2. J. L. Doob, Stochastic processes, Wiley, New York; Chapman and Hall, London, 1953. MR 15, 445.

3. K. Itô, The canonical modification of stochastic processes, J. Math. Soc. Japan 20 (1968), 130-150. MR 37 \#2306.

4. K. Kuratowski, Topologie. Vol. 1, PWN, Warsaw, 1948; English transl., Academic Press, New York; PWN, Warsaw, 1966. MR 19, 873; MR 36 \#440.

5. P. Levy, Théorie de Taddition des variables aléatoires, Gauthier-Villars, Paris, 1954.

6. M. Schilder, Some structure theorems for the symmetric stable laws, Ann. Math. Statist. 41 (1970), 412-421. MR 40 \#8122.

7. E. Hewitt and K. Stromberg, Real and abstract analysis, Springer-Verlag, New York, 1969.

Department of Mathematics, Tulane University, New Orleans, Louisiana 70118

Current address: Department of Mathematics, Sir George Williams University, Montreal, Quebec, Canada 